\title{
Apparatus for Seebeck coefficient and electrical resistivity measurements of bulk thermoelectric materials at high temperature
}

\author{
Zhenhua Zhou and Ctirad Uher \\ Department of Physics, University of Michigan, Ann Arbor, Michigan 48109
}

(Received 9 October 2004; accepted 11 October 2004; published online 5 January 2005)

\begin{abstract}
A high temperature Seebeck coefficient and electrical resistivity measurement apparatus has been designed and built for measuring advanced thermoelectric materials. The apparatus covers the range of temperatures from 300 to $1300 \mathrm{~K}$. Different sources of errors involved in the two measurements are discussed. The accuracy of the electrical resistivity measurement is estimated to be better than $\pm 1 \%$ by measuring standard graphite sample from NIST. (C) 2005 American Institute of Physics.
\end{abstract}

[DOI: $10.1063 / 1.1835631]$

\section{INTRODUCTION}

Thermoelectric materials are considered to be very promising for high-temperature power generation application, especially for harvesting waste heat and for onboard power for deep space missions. Good thermoelectrics can convert heat directly into electrical energy with a reasonable efficiency provided a substantial temperature gradient exists and the material has a high thermoelectric figure of merit. During the past decades most efforts have been concentrated on the improvement of the dimensionless figure of merit (ZT) of thermoelectric materials around room temperature with the aim to develop better thermoelectric coolers. However, for efficient high temperature power generation purpose, good thermoelectric materials with large ZT at high temperatures $(600-1300 \mathrm{~K})$ are highly desirable. In searching for such thermoelectric materials, it is essential to evaluate and therefore measure the following key transport parameters: the Seebeck coefficient, the electrical resistivity, and the thermal conductivity. It is often also useful to have data on the Hall coefficient. In any case, the measurements ought to be done with high accuracy and over a wide range of temperatures so as to fully assess the material in the regime of temperatures where it is intended to operate.

Techniques to measure transport properties at low temperature (from liquid helium temperature up to room temperature) are well covered in the literature, e.g., Refs. 1-6. In comparison, fewer papers dealing with high temperature transport property measurements are available. Almost two decades ago Wood et al. ${ }^{7}$ developed an apparatus for the Seebeck coefficient measurement up to $1900 \mathrm{~K}$ that generates a small temperature gradient with the aid of a light pulse. Later, the same group used an integral method to measure the Seebeck coefficient up to $1000{ }^{\circ}{ }^{8} .^{8}$ A brief mention of the experimental technique also occasionally appears in research papers dealing with high temperature transport studies $^{9,10}$ however, none provide a detailed description of the apparatus nor it states limitations and the sources of measurement errors. In this article we describe an apparatus that was recently setup in our laboratory to measure the Seebeck coefficient and the electrical resistivity to temperatures as high as $1300 \mathrm{~K}$. Different sources of errors involved in the measurements are discussed.

\section{EXPERIMENTAL DETAILS}

\section{A. Description of the apparatus}

Figure 1 shows a schematic diagram of the hardware for the high temperature Seebeck coefficient and electrical resistivity measurement apparatus. Basically, it consists of a well insulated $2 \mathrm{~kW}$ rectangular oven located under a bell jar of a vacuum system pumped with a diffusion pump down to 1 $\times 10^{-6}$ Torr. The oven is constructed from $1 / 4$ in. thick copper panels and is easily accessed by having its front panel attached on hinges. All oven surfaces are watercooled by passing chilled water through $1 / 4$ in. diameter refrigeration copper tubing hard soldered to the panels. The copper tubing on the movable front panel is connected to the rest of the cooling system via 1/4 in. diameter stainless steel flexible tubes. All water-cooling interconnections are facilitated with metal-to-metal Swagelok connectors. The inside of the oven is lined with seven sheets of 0.002 in. thick molybdenum and two $1 / 2$ in. thick layers of alumina insulation. In the inner layer of alumina are machined grooves for coiled nickelchromium alloy high temperature resistive wires that are powered to heat the oven and the sample to the desired temperature.

Inside the oven one can accommodate and instrument either cylindrical or rectangular samples. They are sandwiched between two tungsten rods embedded in high temperature machinable ceramic blocks that in turn are slipped over stainless steel rods that penetrate the sidewalls of the oven. Springs attached to the outside wall of the oven control the pressure of the tungsten rods on the sample. The tungsten rods are $1 \mathrm{in}$. in length, $0.5 \mathrm{in}$. diameter, and are contacted by steel screws threaded through the ceramic collar to provide a path for the electrical current. The current leads, just like all other leads, are brought from the oven via ceramic "spaghetti" and are fastened at the terminals of a small electrical box located on top of the oven. In the two opposing high temperature ceramic blocks are cut fine spiral grooves 


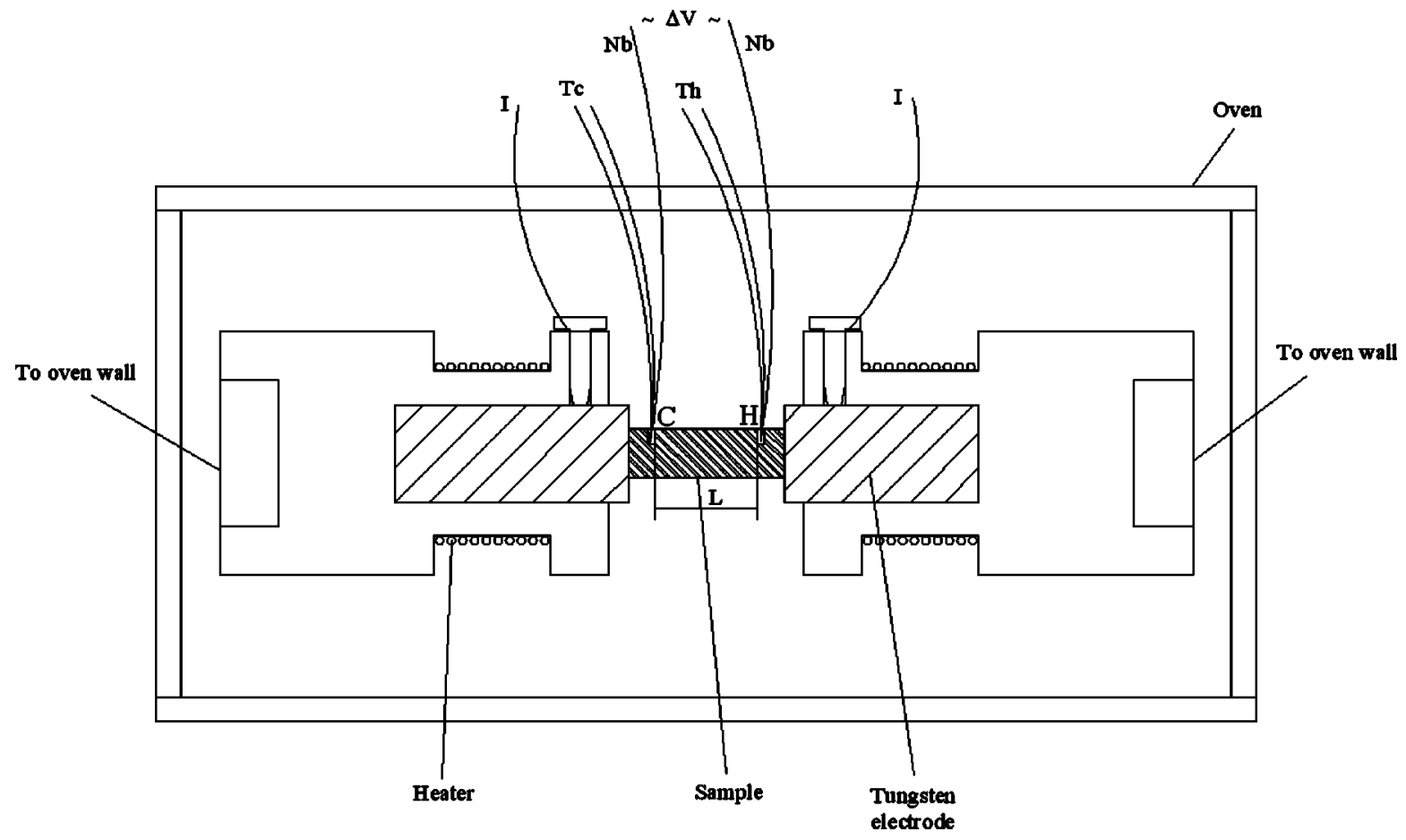

FIG. 1. Schematic diagram of the high temperature Seebeck coefficient and the electrical resistivity measurement apparatus. $L$ is the shortest distance between holes $C$ and $H$.

for resistance heaters that serve to generate a temperature gradient across the sample. The gradient can be applied either from the left- or the right-hand side of the assembly, the feature that provides flexibility as well as the possibility to check the proper functioning of the apparatus. In addition, an independent operation of the two heaters facilitates establishing isothermal conditions for the measurements of electrical resistivity should there be any standing thermal gradients present.

\section{B. Temperature measurement and control}

Accurate temperature setting, its control and measurement are critical to investigations of both the electrical resistivity and the Seebeck coefficient. One needs to know not only the absolute temperature at which the measurement is taken but also the temperature along the length of the sample-hopefully a zero gradient for measurements of electrical resistivity, and an accurately determined gradient when studying the Seebeck effect.

Temperature of the oven is controlled by a Eurotherm 2404 temperature controller that sets and maintains the operational point to within $\pm 0.1{ }^{\circ} \mathrm{C}$ over the range of temperatures extending at least up to $1000{ }^{\circ} \mathrm{C}$. Even when operating at $1000{ }^{\circ} \mathrm{C}$ for a prolonged time, the temperature of the copper panels does not change by more than ten degrees. All temperature sensors are either 0.01 or 0.005 in. diameter K-type chromel-alumel thermocouples from Omega Inc. This type of thermocouple remains stable and is resistant to oxidation at least up to $1000{ }^{\circ} \mathrm{C}$. Its calibration data are reliable and reproducible and the thermocouple signal can be converted directly into temperature values with a resolution of $0.001{ }^{\circ} \mathrm{C}$ using the Keithley 2182 nanovoltmeters. Since the entire oven is under the bell jar in a good vacuum (at
$700{ }^{\circ} \mathrm{C}$ the pressure is typically $5.0 \times 10^{-5}$ Torr), the influence of thermal convection is eliminated and there is negligible temperature drift during the measurement. Typical temperature stability of a sample is better than $\pm 0.05{ }^{\circ} \mathrm{C}$ at $700{ }^{\circ} \mathrm{C}$, and even smaller temperature fluctuation is seen below $700{ }^{\circ} \mathrm{C}$. We make use of the electronic cold junction compensators from Omega Inc. that provide stable reference temperatures $\left(0 \pm 0.2^{\circ} \mathrm{C}\right)$ for the thermocouples.

To attach the thermocouples to the sample, two small holes (0.02-0.04 in.) are drilled along the axial direction of the sample to the depth of about $0.08 \mathrm{in}$. and filled with graphite paste. The thermocouples, together with thin $\mathrm{Nb}$ wires that serve as low thermopower voltage probes, are then inserted into the holes and the sample is heated with a heat gun for a brief period to cure the graphite paste. Temperature gradient along the length of the sample can be set up and controlled with either of the two fine wire heaters wound in the grooves of the ceramic blocks on either side of the sample.

To minimize electromagnetic interference, the junctions of all wires brought from the oven are enclosed in a shielded and thermally isolated box mounted on top of the oven. Thereafter, the wires are brought out from the bell jar using vacuum feedthroughs with the pins made from the same metal as the respective wires.

\section{Electrical resistivity measurement}

Electrical resistivity is measured by a four-probe ac method that eliminates the influence of contact resistance and minimizes the contribution of the thermal emf arising from the Peltier effect. The latter effect is particularly troublesome in thermoelectric materials where the Peltier contribution can lead to serious errors in measurements of 
the electrical resistivity. Since the emf arising from the Peltier effect is of thermal origin, it takes a fraction of a second to develop in the sample. If the excitation current is reversed in a time shorter than the time needed to develop the Peltier-generated emf, the Peltier contribution can be effectively eliminated and a purely ohmic signal can be measured. We use a Linear Research LR-140 bridge with the excitation frequency of $16 \mathrm{~Hz}$ in our measurements of electrical resistivity.

From the measured value of resistance $R$ and sample dimensions (distance between the voltage leads $L$ and the sample cross-section area $S$ ), the electrical resistivity $\rho$ is given by

$$
\rho=R \frac{S}{L} .
$$

Assuming that the sample is in the form of a rectangle, the percentage uncertainty in $\rho$ is given as

$$
\frac{\delta \rho}{\rho}=\frac{\delta R}{R}+\frac{\delta w}{w}+\frac{\delta t}{t}+\frac{\delta L}{L},
$$

where $w$ and $t$ are the width and thickness, $\delta R, \delta w, \delta t$, and $\delta L$ are the uncertainties of $R, w, t$ and $L$, respectively. If the sample is a rod of diameter $D$, then the percentage uncertainty in $\rho$ is

$$
\frac{\delta \rho}{\rho}=\frac{\delta R}{R}+2 \frac{\delta D}{D}+\frac{\delta L}{L} .
$$

There are two distinct error sources in the electrical resistivity measurements. The first one comes from the resistance measurement $\delta R / R$, while the second one originates from the determination of the geometrical factors of the sample, namely $\delta w / w+\delta t / t+\delta L / L$ or $2 \delta D / D+\delta L / L$. Based on the ac resistance bridge specifications, $\delta R / R$ is within $\pm 0.1 \%$. Sample's cross-sectional area $S$ is determined by measuring $w$ and $t$ (or $D$ in the case of a rod) with a micrometer which has an uncertainty of $\pm 0.01 \mathrm{~mm}$. For samples with uniform width and thickness of typically 2 $\times 5 \mathrm{~mm}^{2}, \delta w / w+\delta t / t$, and $2 \delta D / D$ are estimated to be within $\pm 0.4 \%$. $\delta L / L$ is the most uncertain part in Eqs. (2) and (3) for samples with uniform cross section. Usually $L$ is taken as the distance between the centers of the voltage contacts but that will result in an error when the size of the contacts is not a small percentage of the distance between the contacts. In our case, voltage leads are inserted into two small holes drilled into the sample - and as probe separation $L$ we take the shortest distance between the small holes (about $0.5 \mathrm{~mm}$ diameter). The uncertainty in $L$ (measured under the microscope) is within $\pm 0.5 \%$. The total error in measurements of the electrical resistivity is estimated to be within $\pm 1 \%$. However, if the sample is not of uniform cross section, the error in the determination of the cross-section area will be larger than $\pm 0.4 \%$ and the resulting uncertainty in the resistivity measurement may exceed $\pm 1 \%$.

As voltage probes we use thin niobium wires. They serve the purpose of both resistivity measurements as well as measurements of the Seebeck coefficient. The primary advantage of using niobium is its very small and nearly constant absolute thermopower. ${ }^{11}$ For instance, its Seebeck co-

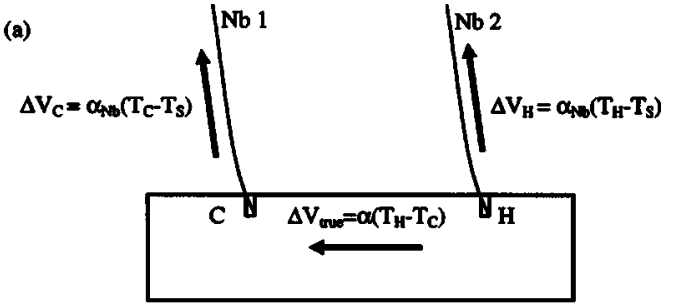

(b)

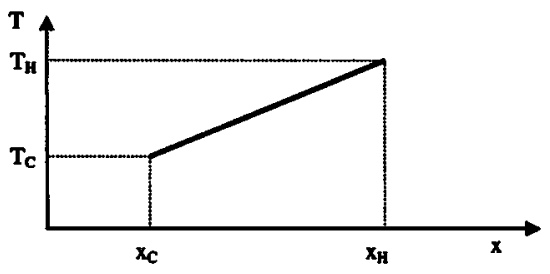

(c)

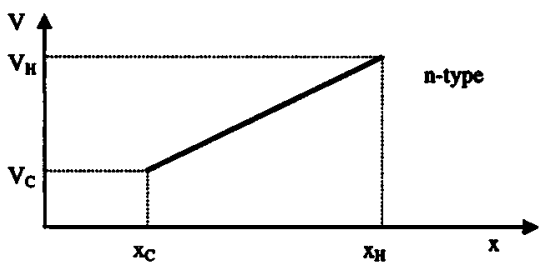

(d)

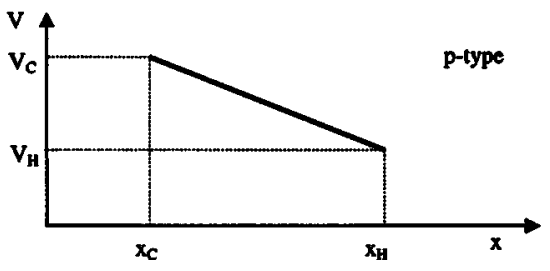

FIG. 2. Principle of the Seebeck coefficient measurement: (a) sample configuration and voltages $\Delta V_{\text {true }}, \Delta V_{C}, \Delta V_{H}$. $T_{S}$ is the environmental temperature. The arrow direction is the positive voltage gradient direction; (b) temperature distribution inside the sample; (c) voltage distribution for $n$-type material; (d) voltage distribution for $p$-type material.

efficient changes from $-2.28 \mu \mathrm{V} / \mathrm{K}$ at $300 \mathrm{~K}$ to $-1.24 \mu \mathrm{V} / \mathrm{K}$ at $1200 \mathrm{~K}$. Moreover, niobium is stable and relatively inert to ambient conditions.

\section{Seebeck coefficient measurement}

Seebeck coefficient, otherwise also known as the thermopower, is usually measured by either a dc or ac technique. ${ }^{12-14}$ The former represents one of the simplest transport measurements. It requires a thermal gradient to be established along a sample and measuring both the thermoelectric voltage and the temperature difference between two points along the length of the sample. The thermopower $\alpha$ is then simply obtained from

$$
\alpha=-\frac{d V}{d T}=-\frac{\Delta V}{\Delta T} .
$$

We base our high temperature measurements on this technique.

Figure 2 shows the principle of the dc Seebeck measuring technique. While a thermal gradient can be generated by heating either end of the sample, for simplicity we assume that heating is applied at the right-hand side of the sample. Figures 2(a)-2(d) conform to this assumption. Thermometers (usually thermocouples or resistive sensors) attached at two points along the length of the sample designate the tempera- 
tures $T_{H}$ and $T_{C}$. The temperature difference between these two points is $\Delta T=T_{H}-T_{C}$. Corresponding to this temperature difference, there will arise a thermoelectric voltage $\Delta V_{\text {true }}$ $=V_{H}-V_{C}$. Depending on the type of a dominant carrier (either electron or hole), the voltage $\Delta V_{\text {true }}$ will be either positive or negative. Specifically, for an $n$-type conductor and the configuration depicted in Fig. 2(c), the sign of $\Delta V_{\text {true }}$ and $\Delta T$ are the same. For a $p$-type conductor such as shown in Fig. 2(d), the sign of $\Delta V_{\text {true }}$ is opposite (negative) to that of $\Delta T$ reflecting the fact that holes diffuse away from the hot towards the cold region of the sample until the thermoelectric voltage arrests their diffusion. It is clear that to measure the Seebeck coefficient accurately, both the temperature and voltage measurements must be accurate.

As already noted, the Seebeck voltage is measured with the aid of thin niobium wires that are inserted into the small holes together with the junctions of the K-type thermocouple. Thus, both the voltage difference $\Delta V_{\text {true }}$ and the temperature difference $\Delta T$ are measured between the same two points $H$ and $C$. The voltage detected at the terminals of the nanovoltmeter is however given by

$$
\Delta V=\Delta V_{\text {true }}+\Delta V_{C}+\Delta V_{H},
$$

where $\Delta V_{C}$ is the voltage generated in the (cold) niobium wire due to the fact that its one end is at the temperature $T_{C}$ while its other end is at the temperature of the nanovoltmeter terminal (presumably a room temperature). Likewise, the voltage $\Delta V_{H}$ arises as a consequence of the hot niobium wire being held between temperatures $T_{H}$ and the temperature of the nanovoltmeter terminal. Since the nanovoltmeter terminals are assumed to be at the same room temperature

$$
\Delta V_{H}+\Delta V_{C}=\alpha_{\mathrm{Nb}}\left(T_{C}-T_{H}\right),
$$

where $\alpha_{\mathrm{Nb}}$ is the Seebeck coefficient of niobium. Therefore, we have the following relation:

$$
\Delta V=\Delta V_{\text {true }}+\alpha_{\mathrm{Nb}}\left(T_{C}-T_{H}\right) .
$$

The Seebeck coefficient is

$$
\alpha=-\frac{\Delta V_{\text {true }}}{\Delta T}=\frac{\Delta V}{T_{C}-T_{H}}-\alpha_{\mathrm{Nb}} .
$$

Thus, the Seebeck coefficient measured with niobium leads is not the absolute Seebeck coefficient of the sample but the Seebeck coefficient relative to niobium. To obtain the sample's absolute Seebeck coefficient the measured $\alpha$ needs to be corrected for the contribution arising from the niobium wires. For most up-to-date thermoelectric materials, their Seebeck coefficients are on the order of $100 \mu \mathrm{V} / \mathrm{K}$ or higher. The contribution of niobium is less than $1 \%$ and therefore negligible.

To ensure the measured Seebeck coefficient falling in the linear range, the temperature difference $\Delta T$ should not exceed a few percents of the absolute sample temperature. In this study the typical temperature difference is $1-8^{\circ} \mathrm{C}$ when the sample temperature is below $1000^{\circ} \mathrm{C}$ so $\Delta T / T$ is usually less than $1 \%$. It is prudent to check the linearity by making measurements with a couple of different $\Delta T$.

The accuracy of the Seebeck coefficient depends on the accuracy of the temperature gradient and the voltage gradi-

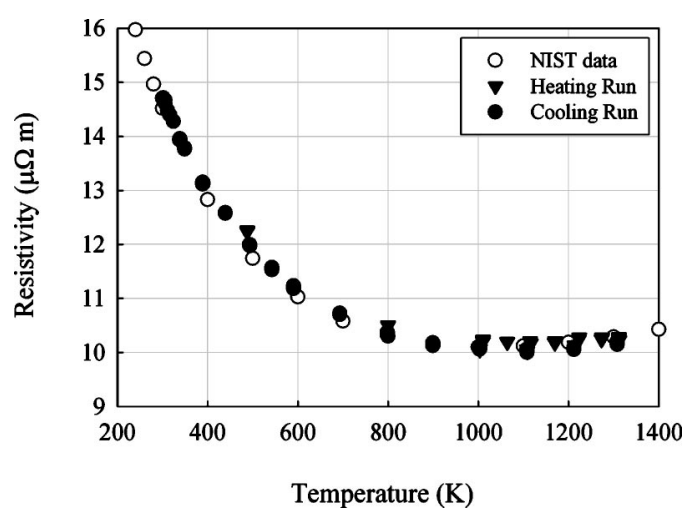

FIG. 3. Electrical resistivity of standard reference material SRM 8424 measured by the present apparatus and the recommended data by NIST.

ent. The accuracy of the temperature gradient measurement is estimated to be within $\pm 0.2 \%$, the accuracy of the voltage gradient measurement is estimated to be within $\pm 0.1 \%$. After taking all these errors into account, the total error of the Seebeck coefficient is estimated to be within $\pm 0.3 \%$.

\section{EXAMPLE OF EXPERIMENTAL RESULTS}

To test our apparatus we have measured the electrical resistivity of graphite standard specimen SRM 8424 purchased from NIST (National Institute of Standards and Technology). The uniform, rod-shaped specimen was $6.46 \mathrm{~mm}$ in diameter (uncertainty less than $\pm 0.01 \mathrm{~mm}$ ) and $20.25 \mathrm{~mm}$ in length. Figure 3 shows our experimental data together with the electrical resistivity data provided by NIST. The resistivity was measured during both heating and cooling runs and the two sets of data are in very good agreement. We estimate the discrepancy between the individual data points does not exceed $\pm 1 \%$. However, if we take the distance between the centers of the drilled holes as $L$ in Eq. (1), the resistivity data will be $10 \%$ smaller than the recommended data from NIST. Therefore, we take the shortest distance between the drilled holes as $L$.

The Seebeck coefficient is very sensitive to the sample composition and microstructures and there is no readily available standard for test.

Instead, in Fig. 4 we show high temperature measurements of the Seebeck coefficient of $\mathrm{Co}\left(\mathrm{Sb}_{1-x} \mathrm{As}_{x}\right)_{3}$ solid solutions with which we work in our search for thermoelectric

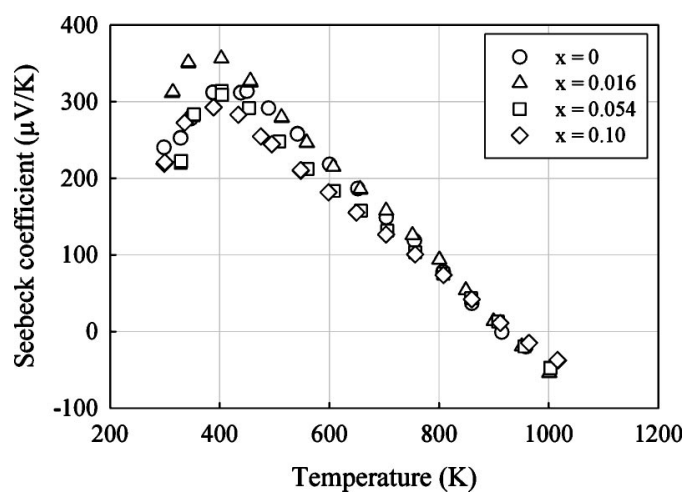

FIG. 4. High temperature Seebeck coefficient of $\mathrm{Co}\left(\mathrm{As}_{x} \mathrm{Sb}_{1-x}\right)_{3}$ measured by the present apparatus. 


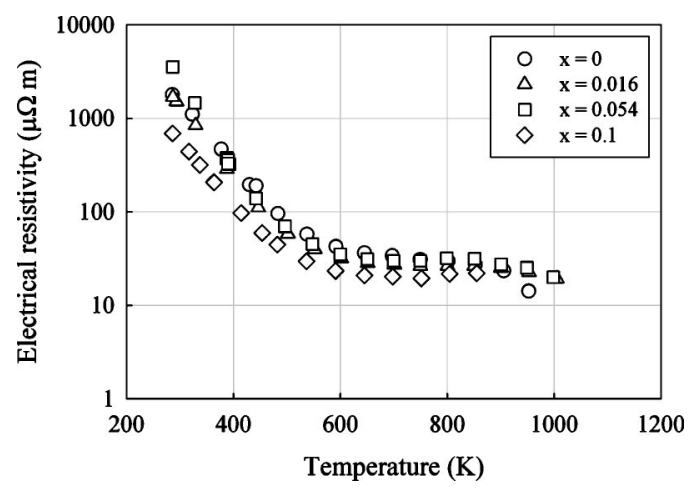

FIG. 5. High temperature electrical resistivity of $\mathrm{Co}\left(\mathrm{As}_{x} \mathrm{Sb}_{1-x}\right)_{3}$ measured by the present apparatus.

materials. Figure 5 depicts the electrical resistivity results of $\mathrm{Co}\left(\mathrm{Sb}_{1-x} \mathrm{As}_{x}\right)_{3}$. Our results show that alloying As with $\mathrm{Sb}$ in the Co-based skutterudite does not change the Seebeck coefficient and the electrical resistivity too much. This is due to the fact that $\mathrm{As}$ and $\mathrm{Sb}$ are isoelectronic and the carrier density is unchanged. However, by forming the As-Sb alloy one dramatically enhances the density of point defects and they very effectively scatter high frequency phonons and thus reduce the overall thermal conductivity. ${ }^{15}$

\section{ACKNOWLEDGMENTS}

The authors thank Dr. T. Caillat and L. D. Zoltan of the Jet Propulsion Laboratory, California Institute of Technology for their kind help and suggestions during the early stage of the development of the high temperature Seebeck and resistivity apparatus. Our research on high temperature thermoelectrics is supported by the Office of Naval Research, Grant No. N00014-03-1-0276.

${ }^{1}$ T. M. Tritt, in Semiconductors and Semimetals, edited by T. M. Tritt (Academic, San Diego, 2001), Vol. 69, p. 25.

${ }^{2}$ C. Uher, Nav. Res. Rev. 48, 48 (1996).

${ }^{3}$ T. M. Tritt, Mater. Res. Soc. Symp. Proc. 478, 25 (1997).

${ }^{4}$ J. Chaussy, A. Guessous, and J. Mazuer, Rev. Sci. Instrum. 52, 1721 (1981).

${ }^{5}$ A. L. Pope, R. T. Littleton IV, and T. M. Tritt, Rev. Sci. Instrum. 72, 3129 (2001).

${ }^{6}$ G. R. Caskey and D. J. Sellmyer, Rev. Sci. Instrum. 40, 1280 (1969).

${ }^{7}$ C. Wood, D. Zoltan, and G. Stapfer, Rev. Sci. Instrum. 56, 719 (1985).

${ }^{8}$ C. Wood, A. Chmielewski, and D. Zoltan, Rev. Sci. Instrum. 59, 951 (1988).

${ }^{9}$ B. A. Cook, J. L. Harringa, S. H. Han, and C. B. Vining, J. Appl. Phys. 78, 5474 (1995)

${ }^{10}$ R. T. Littleton IV, J. Jeffries, M. A. Kaeser, M. Long, and T. M. Tritt, Mater. Res. Soc. Symp. Proc. 545, 137 (1998).

${ }^{11}$ V. Raag and H. V. Kowger, Rev. Sci. Instrum. 36, 2045 (1965).

${ }^{12}$ F. Chen, J. C. Cooley, W. L. Hults, and J. L. Smith, Rev. Sci. Instrum. 72, 4201 (2001).

${ }^{13}$ M. Aubin, H. Ghamlouch, and P. Fournier, Rev. Sci. Instrum. 64, 2938 (1993).

${ }^{14}$ M. A. Howson, M. B. Salamon, T. A. Friedmann, J. P. Rice, and D. Ginsberg, Phys. Rev. B 41, 300 (1990).

${ }^{15}$ C. Uher, Z. Zhou, and T. Caillat, Proceedings of the 23rd International Conference on Thermoelectrics, IEEE Conference Proceedings (in print). 